\title{
Gender differences in the first impressions of autistic adults
}

Running title: Gender and first impressions

Eilidh Cage $^{1}$ and Holly Burton ${ }^{1}$

${ }^{1}$ Royal Holloway, University of London.

Accepted for publication in Autism Research on 26/7/19.

Corresponding author: Dr Eilidh Cage, eilidh.cage@rhul.ac.uk.

Acknowledgements. Thank you to all of the participants involved in this research. Thank you to Paige Mahoney and Maria De Andres for assistance with data collection. Thanks to Noah Sasson for advice. This research was supported financially by the Department of Psychology at Royal Holloway. The authors have no conflicts of interest to declare.

This is the peer reviewed version of the following article: Cage, E. and Burton, H. (2019), Gender Differences in the First Impressions of Autistic Adults. Autism Research, 12: 1495-1504, which has been published in final form a https://doi.org/10.1002/aur.2191 This article may be used for non-commercial purposes in accordance with Wiley Terms and Conditions for self-archiving. 


\begin{abstract}
Prior research has shown that less favourable first impressions are formed of autistic adults by non-autistic observers. Autistic females may present differently to autistic males and could engage in more camouflaging behaviours, which could affect these first impressions. However, research has not yet tested for gender differences in the first impressions of autistic adults. In the current study, non-autistic observers $(n=205)$ viewed either 10 -second video clips or text transcripts in the context of a mock job interview by ten autistic females and ten autistic males, matched to ten non-autistic females and ten non-autistic males. They then rated each stimuli on personality traits (e.g. awkwardness) and behavioural intentions (e.g. "I would start a conversation with this person"). Non-autistic observers were blind to diagnostic status of the individuals in either modality. Results showed that first impressions were less favourable overall of autistic adults in the video modality. Further, autistic females were rated more favourably than autistic males in the video modality across most traits - but autistic females were also rated less favourably than both non-autistic females and males. Some judgements were also made in the text modality, whereby more favourable first impressions were made of autistic males on the basis of speech content. Understanding the first impressions that both autistic females and males make has important implications for diagnostic services and employment prospects.
\end{abstract}

Key words: first impressions; social evaluation; autistic adults; gender differences. 


\section{Lay Summary}

We found that non-autistic people formed more negative first impressions of autistic people, and this was influenced by gender of the person being evaluated. Autistic women were judged more favourably than autistic men, however, both autistic women and men were rated less positively than non-autistic people, with large differences between judgements of autistic females in comparison to non-autistic females. The findings have implications for clinicians and employers who may make rapid judgements based on someone's gender. 


\section{Introduction}

Autism is a neurodevelopmental condition characterised by differences in social communication, social interactions, focused interests, repetitive behaviours and sensory sensitivities (American Psychological Association (APA), 2013). Understanding how autistic people are perceived by non-autistic people has implications for diagnostic services, employment prospects and the general inclusion of autistic people into society. In a series of studies, Sasson et al. (2017) found that first impressions of autistic people were poorer than those of non-autistic people. First impressions are social judgements made in the initial moments of interaction (Wood, Pugh, Touchie, Chan, \& Humphrey-Murto, 2018) and form quickly using "thin slices" of information (Ambady \& Rosenthal, 1992). First impressions can influence behavioural responses and social interactions (Ambady, Bernieri, \& Richeson, 2000). Autistic adults report having fewer reciprocated social relationships than non-autistic individuals (Human, Sandstorm, Biesanz, \& Dunn, 2012) which may relate to negative first impressions (Sasson et al., 2017). As gender differences are noted in the social behaviour of autistic adults (Levitt, 2017), it is possible that first impressions may be affected by the gender of the person being evaluated, although research has not yet explored this.

Accordingly, it is vital that the first impressions of autistic females are understood, as the perception of autistic females by others - such as clinicians or employers - could impact on outcomes for autistic women. Thus far, research has indicated that first impressions formed of autistic adults (irrespective of gender) are worse than the impressions formed of nonautistic adults (e.g. Sasson et al., 2017; Sasson \& Morrison, 2019). Research using vignettes describing autistic characteristics notes that non-autistic observers have stronger intention to distance themselves (Butler \& Gillis, 2011) and display fewer positive attitudes towards autistic adults (Matthews, Ly, \& Goldberg, 2015). Interestingly, this preference for non- 
autistic adults over autistic adults is also observed in autistic observers (Grossman, Mertens \& Zane, 2018). Similar patterns are displayed towards autistic children, who are rated as more socially awkward (Grossman, 2015) and as having fewer friends (Sasson et al., 2017, Study Three).

Sasson et al. (2017) examined first impression formation using a range of experimental studies, one of which the current study aims to partly replicate. Sasson et al. (2017, Study One) used the High-Risk Social Challenge (HiSoC; Gibson, Penn, Pristein, Perkins, \& Belger, 2010) where participants engage in a mock audition for a reality TV show. The HiSoC was completed by autistic (17 male, three female) and non-autistic (17 male, three female) adults in Sasson et al.'s (2017) first experiment to produce 10 seconds of stimuli, which was adapted into different presentation modalities (e.g. video and audio). Nonautistic observers then rated the stimuli in terms of traits (e.g. awkwardness) and behavioural intentions (e.g. hanging out with the person). Autistic adults in the video modality were perceived as more awkward, submissive, and less likeable and attractive than matched nonautistic adults by non-autistic observers unaware of diagnostic status.

In their experiment, only three autistic females and three non-autistic females were used as stimuli, with no analysis of gender differences in first impressions possible (Sasson et al., 2017, Study One). It might be hypothesised that gender impacts on first impressions, such that autistic females may be evaluated more positively than autistic males. The ratio of autistic males to females is estimated at 3:1 males to females (Loomes, Hull, \& Mandy, 2017), a reduction in the previous ratio of $4: 1$. Loomes et al. (2017) speculate this change may be due to under-diagnosis and misdiagnosis of autism in females, aspects which could relate to first impressions made by clinicians. Underlying these diagnostic issues could be the finding that autistic females may be more socially motivated than autistic males (Sedgewick 
et al., 2016) and could differ in their presentation of core diagnostic markers of autism (Hull \& Mandy, 2017).

Further, there may be more camouflaging in autistic females (Hull \& Mandy, 2017). Camouflaging involves learning how to act in social situations (Lai et al., 2011) and is employed as a social strategy by autistic people to conceal social difficulties and navigate social situations (Hull et al., 2017). These strategies can involve mimicking behaviour, conversation style and mannerisms of non-autistic peers (Lai et al., 2011). Some studies find camouflaging is more prevalent in females (Lai et al., 2011; Sasson \& Morrison, 2019), while others report no gender differences in camouflaging (Cage et al., 2018; Hull et al., 2017) or that gender differences in camouflaging are driven by societal expectations of autistic women (Cage \& Troxell-Whitman, 2019). One might hypothesise that camouflaging could have an impact on first impressions (Sasson et al., 2017); if camouflaging is more prevalent in autistic females, this may be reflected in more favourable first impressions.

The current study therefore aimed to partially replicate Sasson et al.'s (2017, Study One) findings and extend these findings by examining differences in the first impressions formed of autistic males and females. Accordingly, an equal number of autistic females and autistic males were matched to non-autistic females and non-autistic males for the creation of stimuli. Non-autistic observers either watched 10 -second video clips of these individuals or read transcripts of the speech content of these 10-seconds. Gender of the observer was controlled for, since observer gender may bias how they perceive others of the same or opposite gender (Mattarozzi et al., 2015; Rudman \& Goodwin, 2004). Further, the current study extends Sasson et al.'s (2017, Study One) ecological validity by using a mock job interview task, rather than the HiSoC task. The mock job interview task allows for first impressions to be explored in an employment context and presents a situation which is more likely to be 
encountered in everyday life. Testing first impressions in an employment context is important given the employment difficulties faced by autistic people (Taylor \& Seltzer, 2010), with autistic adults reporting poor job retention (Halladay et al., 2015) despite the possession of relevant skills, training, and education (Friedman, Warfield \& Parish, 2013). Based on the literature, it is hypothesised that overall, autistic adults will be rated less favourably than nonautistic adults in the video modality. Second, it is anticipated that autistic females will be rated more favourably than autistic males in the video modality. 


\section{Methods}

\section{Participants}

\section{Stimuli participants}

In total, 40 stimuli participants (20 autistic, 20 non-autistic) were recruited through previous contacts, online advertisements and university student support services. Participants were matched on gender (10 males and 10 females per group); age (autistic mean $=22.75(S D=$ $3.70)$, non-autistic mean $=23.35(S D=4.61) ; p=.65)$; verbal comprehension $($ autistic mean $=$ $115.45(S D=16.14)$, non-autistic mean $=117.70(S D=13.77) ; p=.64) ;$ and full intellectual ability $($ autistic mean $=114.35(S D=13.53)$, non-autistic mean $=116.80(S D=13.27) ; p=$ .58), as measured by the Wechsler Abbreviated Scale of Intelligence-II (WASI-II; Weschler, 2013). All participants were of White ethnicity. All autistic participants reported a diagnosis of an Autism Spectrum Condition, with a mean age of diagnosis at 14.89 years $(S D=6.40)$. All participants completed the Ritvo Autism Aspergers Diagnostic Scale (RAADS-14; Eriksson, Andersen \& Bejerot, 2013) to confirm autism diagnoses (autistic mean $=31.55$ (SD $=6.68)$, non-autistic mean $=5.8(S D=4.76) ; p<.001)$, with all autistic participants scoring above the cut-off of 14 and no non-autistic participant scoring above it. There was a significant interaction between gender and group for RAADS scores $(\mathrm{F}(1,36)=4.30, p$ $\left.=.045, \eta^{2} p=.11\right)$, such that autistic females $(\mathrm{M}=34.90, S D=4.91)$ had higher RAADS scores than autistic males $(\mathrm{M}=28.20, S D=6.73 ; p=.02)$.

\section{Experiment participants}

Observers were recruited from Royal Holloway, University of London, where they were approached on campus facilities, through word-of-mouth, or participated as first-year Psychology student for course credit. The sample consisted of 205 participants who were 
randomly assigned to either the video $(n=109)$ or text $(n=96)$ modality condition. This sample size is similar to that obtained by Sasson et al. (2017, Study One). The sample consisted of 167 females $(81.5 \%)$ and 38 males $(18.5 \%)$ with a mean age of $20.58(S D=$ 3.96). In the video modality there were 89 females (81.7\%) and 20 males (18.3\%) with a mean age of $20.46(S D=3.61)$, and in the text modality there were 78 females $(81.3 \%)$ and 18 males $(18.8 \%)$ with a mean age of $20.71(S D=4.32)$. Gender of observer was controlled for in all analyses. 28 participants $(13.7 \%)$ reported that they had a family member with an autism diagnosis, and none had a diagnosis of autism themselves. Further demographic information to characterise the sample can be seen in Table 1, demonstrating that there was a predominantly White student sample.

Ethical approval was granted via the ethical procedure at Royal Holloway, University of London and all participants gave full informed consent before participating.

[Insert Table 1 here]

\section{Materials and procedure}

\section{Stimuli creation}

Participants completed a task which involved the completion of an imaginary scenario; a mock interview for their dream job. A digital camera was positioned on a tripod to record the participant from the shoulders upward in front of a white background. In the mock interview, participants were asked to describe their dream job before answering two questions: "Tell me about yourself and why you want this job" and "Do you have any qualities that you think make you suitable for this job?" Two questions were asked to ensure ethically suitable responses as some participants disclosed personal information (e.g. name, University) when 
the first question was asked. After videotaping, the WASI-II, RAADS-14 and a demographic questionnaire was completed.

Each videotaped scenario provided around 45 to 60 seconds of social presentation and was edited to a 10-second clip of the participant's initial response to either question to be included as the stimuli. 10-seconds was deemed an appropriate length providing enough evidence of social behaviour to make a reliable first impression whilst providing sufficient audio to transcribe and include in the text modality condition (Sasson et al., 2017), as well as providing experimental consistency in stimuli length. Effort was made to ensure that participant's speech was not cut off, however this was not possible for all participants thus some responses ended mid-phrase. All 10-second video clips were transcribed in verbatim for the text modality condition, and all stimuli were presented in a random order using the Qualtrics survey platform.

\section{Measure of Traits and Behavioural Intentions}

Items were patterned after those used by Sasson et al. (2017, Study One) to assess perceptions of different characteristics. Originally, this comprised of 10 items, including six personality traits (i.e., "This person is socially awkward", "This person is attractive") and four behavioural intent items (i.e., "I would hang out with this person in my free time", "I would start a conversation with this person"). An additional item ("I would employ this person") was added in the current study to examine perceived employability. All items were randomised and rated using a four-point scale indicating level of agreement with each statement $(1=$ Strongly Agree, 4 = Strongly Disagree $)$. Prior to analysis, items "I would mind if I had to talk to this person", "I would be uncomfortable sitting next to this person" and "this person is socially awkward" were reverse coded so that higher scores indicated more positive judgements. 


\section{Experimental Procedure}

Observers completed the study on a desktop computer. The experiment was randomised to ensure participants observed and rated only one modality (videos or text) for each of the 40 stimuli participants. Observers were told that the video clips or text were from different people who had been asked to think about what they would say if they were in an interview for their dream job. Observers were blind to the diagnosis of the stimuli participants, and in the text modality were also blind to the gender, only reading the transcript of speech content. After each stimuli, observers rated each stimuli on personality traits and behavioural intentions. Once all stimuli had been rated, participants completed a brief demographic questionnaire. Each session lasted up to 45 minutes.

\section{Design and data analysis}

This study had an experimental design. A two (group: autistic or non-autistic) by two (gender of stimuli: male or female) by two (modality: video or text) by 11 (traits) mixed ANCOVA controlling for observer gender was conducted on the mean trait ratings. Gender was entered as a covariate in the analysis since the gender of the observer could influence perceptions. In post-hoc analyses examining trait comparisons, a corrected alpha level of $p<.0045$ is used to take into account multiple comparisons. 


\section{Results}

A two (group: autistic or non-autistic) by two (gender of stimuli: male or female) by two (modality: video or text) by 11 (traits) mixed ANCOVA controlling for observer gender was used to examine differences in first impressions. Mauchly's Test of Sphericity indicated that the assumption of sphericity had been violated $(p<.001)$, therefore Greenhouse-Geisser estimates were utilised.

There was a significant main effect of traits $\left(\mathrm{F}(4.67,943.18)=6.49, p<.001, \eta p^{2}=.031\right)$ indicating differences within the traits regardless of group, gender or mode. There was a significant main effect of modality $\left(\mathrm{F}(1,202)=4.48, p=.036, \eta p^{2}=.022\right)$ such that ratings were higher in the text modality $(M=2.72)$ than video modality $(M=2.66)$. No other main effects were significant ( $p s>.68)$.

There was a significant interaction between group and modality $(\mathrm{F}(1,202)=271.99, p<.001$, $\left.\eta p^{2}=.57\right)$. Pairwise comparisons using Bonferroni to adjust for multiple comparisons showed no significant difference between groups in the text modality overall $(p=.16)$, but there was a significant difference between groups in the video modality, such that overall autistic people were rated less favourably $(M=2.52)$ than non-autistic people $(M=2.80, p<.001)$. There was also a significant interaction between stimuli gender and modality $(\mathrm{F}(1,202)=71.48, p<.001$, $\left.\eta p^{2}=.26\right)$. Pairwise comparisons showed only a significant difference between genders in the video modality, such that females overall were rated more favourably $(\mathrm{M}=2.73)$ than males $(\mathrm{M}=2.59 ; p<.001)$. There was a significant two-way interaction between traits and group $\left(\mathrm{F}(6.51,1314.37)=4.19, p<.001, \eta p^{2}=.02\right)$ and between traits and mode $(\mathrm{F}(4.67,943.18)=$ $\left.10.43, p<.001, \eta p^{2}=.049\right)$. Since these interactions are qualified by subsequent three- and four-way interactions they are not broken down here. No other two-way interactions were significant. 
There was a significant three-way interaction between traits, group and modality $(\mathrm{F}(6.51$, $\left.1314.37)=45.06, p<.001, \eta p^{2}=.18\right)$, traits, stimuli gender and modality $(\mathrm{F}(6.67,1347.85)=$ $\left.13.02, p<.001, \eta p^{2}=.061\right)$ and traits, group and stimuli gender $(\mathrm{F}(7.24,1464.02)=2.08$, $\left.p=.04, \eta p^{2}=.01\right)$. These three-way interactions were qualified by a four-way interaction between traits, group, stimuli gender and modality $(\mathrm{F}(7.25,1464.02)=12.15, p<.001$, $\eta p^{2}=.057$. All other three-way interactions were not significant.

For the four-way interaction between traits, group, stimuli gender and modality, pairwise comparisons using Bonferroni to correct for multiple comparisons were used to examine the interaction (corrected alpha level of $p<.0045$ ). Effect sizes were calculated following the guidelines of Lakens (2013). In the video modality (Figure 1), comparing between groups, there were significant differences between autistic and non-autistic females across all of the traits apart from 'smart' ( $\left.p=.08, d_{z}=.15\right)$. Specifically, non-autistic females were rated more favourably than autistic females for awkwardness $\left(p<.001, d_{z}=2.16\right)$, attractiveness $(p<.001$, $\left.d_{z}=1.65\right)$, 'employ' $\left(p<.001, d_{z}=1.35\right)$, likeable $\left(p<.001, d_{z}=1.24\right)$, 'hang out' $\left(p<.001, d_{z}\right.$ $=1.15)$, 'start conversation' $\left(p<.001, d_{z}=1.06\right)$, 'sit next to' $\left(p<.001, d_{z}=.85\right)$, 'live near' $\left(p<.001, d_{z}=.56\right)$, dominant $\left(p<.001, d_{z}=.37\right)$ and trust $\left(p<.002, d_{z}=.27\right)$.

There were significant differences between males according to group in the video modality non-autistic males were rated more favourably than autistic males for awkwardness $(p<.001$, $\left.d_{z}=1.46\right)$, attractiveness $\left(p<.001, d_{z}=1.18\right)$, 'employ' $\left(p<.001, d_{z}=1.04\right)$, 'hang out' $(p<.001$, $\left.d_{z}=.93\right)$, 'start conversation' $\left(p<.001, d_{z}=.83\right)$, 'sit next to' $\left(p<.001, d_{z}=.73\right)$, likeable $\left(p<.001, d_{z}=.69\right),{ }^{\prime}$ live near' $\left(p<.001, d_{z}=.52\right)$, smart $\left(p<.001, d_{z}=.45\right)$ and dominant $(p<.001$, $\left.d_{z}=.35\right)$. Trust was the only trait not significantly different when considering the corrected alpha level of $.0045\left(p=.006, d_{z}=.27\right)$. 
Further, when comparing within groups by gender, there were significant differences in the video modality - autistic females were rated more favourably than autistic males for attractiveness $\left(p<.001, d_{z}=.68\right)$, trust $\left(p<.001, d_{z}=.56\right)$, 'live near' $\left(p<.001, d_{z}=.55\right)$, 'sit next to' $\left(p<.001, d_{z}=.52\right)$, 'start conversation' $\left(p<.001, d_{z}=.48\right)$, smart $\left(p<.001, d_{z}=.46\right)$, 'hang out' $\left(p<.001, d_{z}=.44\right)$ and likeable $\left(p=.001, d_{z}=.31\right)$. Autistic males were rated as more dominant than autistic females $\left(p<.001, d_{z}=.41\right)$. Considering the corrected alpha level of .0045 there was no significant difference in awkwardness ( $\left.p=.04, d_{z}=.19\right)$ or for 'employ' $\left(p=.92, d_{z}=.009\right)$.

Non-autistic females were rated more favourably than non-autistic males in the video modality for awkwardness $\left(p<.001, d_{z}=1.18\right)$, attractiveness $\left(p<.001, d_{z}=1.15\right)$, likeable ( $\left.p<.001, d_{z}=1.06\right)$, 'hang out' $\left(p<.001, d_{z}=1.00\right)$, 'start conversation' $\left(p<.001, d_{z}=.99\right)$, 'sit next to' $\left(p<.001, d_{z}=.72\right)$, 'live near' $\left(p<.001, d_{z}=.60\right)$, trust $\left(p<.001, d_{z}=.54\right)$ and 'employ' ( $\left.p<.001, d_{z}=.40\right)$. With the corrected alpha level, there were no significant differences between non-autistic females and males for smart $\left(p=.020, d_{z}=.23\right)$ or dominant $\left(p=.057, d_{z}\right.$ $=.17)$.

Differences across gender and group (for example, to compare autistic females to non-autistic males) were considered. In the video modality, autistic females were rated less favourably than non-autistic males for awkwardness ( $\left.p<.001, d_{z}=1.65\right)$, 'employ' $\left(p<.001, d_{z}=.95\right)$, dominant $\left(p<.001, d_{z}=.70\right)$, attractiveness $\left(p<.001, d_{z}=.46\right)$, likeable $\left(p<.001, d_{z}=.37\right)$, 'hang out' ( $\left.p=.001, d_{z}=.32\right)$ and 'start conversation' $\left(p=.002, d_{z}=.30\right)$. There were no significant differences for trust $\left(p=.029, d_{z}=.21\right)$, 'sit next to' $\left(p=.073, d_{z}=.17\right)$, 'live near' $\left(p=.44, d_{z}\right.$ $=.07)$ or smart $\left(p=.81, d_{z}=.023\right)$.

Autistic males were rated less favourably than non-autistic females for awkwardness $(p<.001$, $\left.d_{z}=2.04\right)$, attractiveness $\left(p<.001, d_{z}=1.83\right)$, 'hang out' $\left(p<.001, d_{z}=1.36\right)$, likeable $(p<.001$, 
$\left.d_{z}=1.27\right)$, 'start conversation ( $\left.p<.001, d_{z}=1.21\right)$, 'employ' $\left(p<.001, d_{z}=1.17\right)$, 'sit next to' $\left(p<.001, d_{z}=.97\right)$, 'live near' $\left(p<.001, d_{z}=.83\right)$ and trust $\left(p<.001, d_{z}=.67\right)$. There was no significant difference for 'smart' $\left(p=.045, d_{z}=.19\right)$ or dominant ( $\left.p=.33, d_{z}=.093\right)$.

[Insert Figure 1 here]

Within the text modality (Figure 2), there were significant differences between and within groups. First, comparing between groups, autistic females were rated more favourably than non-autistic females in the text modality only for dominant $\left(p=.004, d_{z}=.33\right)$. There were significant differences between autistic and non-autistic males, with the direction of effect such that autistic males were rated more favourably then non-autistic males for smart $\left(p<.001, d_{z}=.57\right)$, 'hang out' $\left(p<.001, d_{z}=.45\right)$, 'live near' $\left(p<.001, d_{z}=.39\right)$ and 'start conversation' $\left(p=.001, d_{z}=.39\right)$.

Comparing between genders in the text modality, autistic males were rated more favourably than autistic females for 'live near' ( $\left.p<.001, d_{z}=.43\right)$, 'employ' $\left(p=.001, d_{z}=.34\right)$ and smart $\left(p=.002, d_{z}=.31\right)$. Non-autistic females were rated more favourably than non-autistic males for 'hang out' $\left(p<.001, d_{z}=.47\right)$, likeable $\left(p<.001, d_{z}=.47\right)$, 'start conversation' $\left(p<.001, d_{z}\right.$ $=.43$ ) and awkwardness $\left(p=.002, d_{z}=.33\right)$; whereas non-autistic males were rated more favourably than non-autistic females for the trait 'dominant' $\left(p<.001, d_{z}=.39\right)$.

Comparing autistic females to non-autistic males, in the text modality autistic females were rated more favourably for 'smart' $\left(p<.002, d_{z}=.31\right)$ and all other comparisons were not significant. Similarly, autistic males were rated more favourably than non-autistic females for 'smart' ( $\left.p=.001, d_{z}=.34\right)$. All other comparisons were not significant at the corrected alpha level. 


\section{Discussion}

The current study provides novel insight into how autistic males and females are perceived in comparison to non-autistic males and females. This study expands on Sasson et al.'s (2017, Study One) findings by enabling comparisons between genders and finds significant differences in first impressions depending on gender and group. First, autistic people were rated less favourably overall than non-autistic people, replicating Sasson et al.'s (2017) earlier work and others (e.g. Matthews et al., 2015). The implications of these overall poorer first impressions are considered below. Second, after taking into account the observer's own gender, in the video modality autistic females were evaluated less positively than non-autistic females across all traits except 'smart'. Largest effect sizes were noted for awkwardness, employability, likeability, starting a conversation or hanging out with the individual. Autistic males were also evaluated less positively than non-autistic males in the video modality across all traits except smart, with largest effect sizes for awkwardness, attractiveness and employability. When considering within-group differences, in the video modality autistic females were rated more positively than autistic males across eight out of eleven traits and seen as significantly less dominant, with medium sized effect sizes overall.

These findings imply that the differences were greater between first impressions of autistic females in comparison to non-autistic females than between autistic females and autistic males. In the video modality, non-autistic females were rated more positively than nonautistic males for nine out of eleven traits, with large effect sizes for awkwardness, attractiveness, likeability, hanging out and starting a conversation. Comparing across gender and group, autistic females were rated less favourably then non-autistic males for six out of eleven traits in the video modality, with largest effect sizes for awkwardness and employability. Autistic males were also rated more negatively than non-autistic females for 
nine out of eleven traits, with largest effect sizes for awkwardness, attractiveness, hanging out, likeability, starting a conversation and employability.

Negative first impressions may reduce the willingness of a social partner to pursue and progress a social relationship (Bromgard \& Stephan, 2006), evoke avoidance behaviours (Blascovitch, Mendes, Hunter \& Lickel, 2000) and 'cold' responses (Wood et al., 2018). These findings pose significant implications for autistic people. Autistic individuals often experience bullying, victimisation (Cappadocia, Weiss \& Pepler, 2012) and stigmatisation (Shtayermman, 2009). The reduced intent to pursue social interaction with autistic adults could contribute to these experiences (Jones, Pickles, \& Lord, 2017; Matthews, Ly, \& Goldberg, 2015) and may affect psychological wellbeing due to a perceived lack of autism acceptance (Cage et al., 2018). The negative rating of autistic people provides support for the ‘double empathy problem’ (Milton, 2012) which argues that non-autistic individuals lack empathy into the lives of autistic people, and a breakdown of reciprocity in social interactions between autistic and non-autistic individuals is observed (Milton \& Bracher, 2013). The current findings support the idea that difficulties in social interactions between autistic people and non-autistic people are not purely a result of the autistic person's social difficulties, but also a product of the rapid unfavourable judgements made by non-autistic individuals (Milton, Heasman \& Sheppard, 2018).

The current study makes a unique contribution in understanding how gender influences first impressions. Although autistic females were rated more favourably than autistic males across most traits, they were rated less favourably than non-autistic females and non-autistic males across numerous traits. Whilst prior research has reported no gender differences in camouflaging behaviour (Cage et al., 2018; Hull et al., 2017), Lai et al. (2016) argue that autistic females may camouflage with greater success than autistic males. However, the 
current findings do not necessarily support this hypothesis. Rather, they suggest that autistic females do have a different social presentation to autistic males, and since non-autistic females were also rated more positively than non-autistic males, there could be a 'protective female effect' rather than camouflaging. This protective effect may relate to socialisation or biological differences (Hyde, 2014) that prompt the perceiver to view females more positively. Although males and females are more similar than they are different on psychological variables, Hyde (2005) discusses how assumptions are often made about gender which impacts on outcomes, from the workplace to relationships. Gendered expectations could bias the perceptions of the social abilities of autistic individuals, which may further relate to camouflaging (Dean, Harwood \& Kasari, 2016). Simply presenting as female could promote positive first impressions, but perceivers are still sensitive to autistic differences in social presentation.

Interestingly, autistic females in our sample had higher RAADS scores than autistic males, indicative of more autistic characteristics. Despite this, the autistic females were perceived more positively than autistic males. Thus, we cannot rule out that autistic females were camouflaging their autistic characteristics to a greater extent. It should be noted, however, that the current study did not measure the camouflaging strategies of the stimuli participants, which future research should do to further test camouflaging efficacy. Since autistic females were still negatively judged in comparison to non-autistic females and males, any camouflaging strategies undertaken by autistic females do not necessarily translate into more positive first impressions. It may also be the case that autistic males camouflage but are not as skilled in doing so, which could contribute to more negative first impressions. Nonetheless, in terms of effect sizes, some of the biggest differences were noted between autistic females and non-autistic females, suggesting that negative first impressions of autistic females remain to be substantial. 
Interestingly, given that the experimental paradigm focused on an employment context, autistic males and females were rated as less employable than non-autistic participants in the video modality. These findings follow prior research stating that autistic individuals find it challenging to obtain employment (Engström et al., 2003). Statistics show that with increasing qualification level, employment outcomes for autistic people are worse (Allen \& Coney, 2018). Social presentation is typically not researched when assessing employment in autism (Schaller \& Yang, 2005), and the current findings may suggest that poor employment outcomes could relate to the formation of initial negative first impressions by employers (Hendricks, 2010). Indeed, Hurlbutt and Chalmers (2004) report that autistic adults struggle in interviews, which may relate to negative first impressions. However, research is needed with observers who make decisions about employing people (e.g. managers or those in Human Resources) to test whether the current findings apply in real employment contexts. Interestingly, autistic males were rated more favourably within the text modality for some traits in comparison to non-autistic males, non-autistic females and autistic females. This finding may suggest that negative first impressions are driven by social presentational style rather than substantive speech content (Sasson et al., 2017; Zaki, 2003). As autistic males may be subject to greater vetting in interviews due to higher societal expectations (Powers, Hogansen, Geenen, Powers, \& Gil-Kashiwabara, 2008), it is important employers are mindful of how first impressions might influence their willingness to employ. However, the findings within the text modality have small effect sizes and as such are interpreted with caution.

The observed gender differences in first impressions also have clinical implications, since autistic females were perceived more positively than autistic males in the video modality. Clinicians should be aware of rapid judgements and biases in perceiving females more positively. Autistic females may be at greater risk of not receiving a timely diagnosis 
(Loomes et al., 2017) therefore the gendered perceptions of clinicians could play a role in preventing access to diagnosis. Access to diagnosis for all from an early age - irrespective of gender - is vital to ensure appropriate support is put in place. However, educational support systems, such as inclusive practices, still have a long way to go in terms of providing sufficient support for autistic children (Pellicano, Bolte, \& Stahmer, 2018).

Further, it is vital that research continues to examine how non-autistic perceptions of autistic people can be changed, as these perceptions could be a barrier to effective inclusion. Sasson and Morrison (2019) found that labelling stimuli as autistic improved first impressions. They also found that greater autism knowledge was associated with more positive first impressions. Such findings suggest that education may be important to help non-autistic peers grasp the diverse features of autism and both the challenges and strengths experienced by autistic people. Indeed, an educational intervention for university students with such features improved knowledge and decreased stigmatisation of autism (Gillespie-Lynch et al., 2015). Alongside the current findings, we suggest such educational interventions tackle gendered stereotypes of autism.

\section{Limitations and future directions}

The current study is limited by a student observer sample with mostly female participants. However, this sample is similar to that recruited by Sasson et al. (2017, Study One) which demonstrates replicability within a female student sample. Future research is needed with more diverse samples as well as with further consideration of the individual differences that may contribute to first impressions (Morrison, DeBrabander, Faso, \& Sasson, 2019). It is also important to examine how first impressions might differ according to race, since the current study included only White participants in the stimuli. Further, the sample is limited by a student sample making decisions about employability, furthering the need for more research 
with different groups. We also did not use a fine-grained measure of past experience with autistic people, which could also be a variable that impacts on first impressions of autism (Morrison et al., 2019).

Additionally, stimuli were obtained from social presentations in a mock job interview, which could have lacked ecological validity. Stimuli participants were aware they were taking part in an experiment rather than a real interview; therefore, their behaviour may not reflect realworld behaviour. Given the difficulties experienced by autistic individuals when seeking employment (Halladay et al., 2015) it is important that research is conducted to tackle barriers that may be affecting employment prospects, including and beyond first impressions. Additionally, verification of autism diagnosis was only possible via a screening measure rather than a full diagnostic assessment for research purposes (e.g. ADOS, Lord et al., 2000), therefore a degree of caution is warranted in interpretation of the current findings.

Despite these limitations, this study adds to evidence that less favourable first impressions are formed of autistic adults than non-autistic adults and contributes to current understandings of autistic adults in terms of employability and diagnosis. These findings suggest the negative nature of first impressions could contribute to the reduced quality and quantity of social interactions between autistic and non-autistic adults (Sasson et al., 2017) and demonstrate that although autistic females were rated more positively than autistic males, first impressions are still more negative for both genders in comparison to first impressions of non-autistic people. The first impressions made by non-autistic people could serve as a potential barrier to timely diagnosis and equal rights to employment. 


\section{References}

Allen, M. \& Coney, K. (2018). What happens next? 2018 A report on the first destinations of 2016 disabled graduates. Retrieved from https://www.agcas.org.uk/KnowledgeCentre/7991a7d5-84a0-4fe1-bbdc-5313d9039486.

Ambady, N., \& Rosenthal, R. (1992). Thin slices of expressive behavior as predictors of interpersonal consequences: A meta-analysis. Psychological Bulletin, 111(2), 256.

Ambady, N., Bernieri, F. J., \& Richeson, J. A. (2000). Toward a histology of social behavior: Judgmental accuracy from thin slices of the behavioral stream. Advances in Experimental Social Psychology, 32, 201-271.

American Psychological Association. (2013). Diagnostic and Statistical Manual of Mental Health Disorders (5 ${ }^{\text {th }}$ ed.). Arlington, VA: American Psychiatric Publishing.

Blascovich, J., Mendes, W. B., Hunter, S. B., \& Lickel, B. (2000). Stigma, threat, and social interactions. The social psychology of stigma, 307-333.

Bromgard, G., \& Stephan, W. G. (2006). Responses to the stigmatized: Disjunctions in affect, cognitions, and behavior. Journal of Applied Social Psychology, 36(10), 2436-2448.

Butler, R. C., \& Gillis, J. M. (2011). The impact of labels and behaviors on the stigmatization of adults with Asperger's disorder. Journal of autism and developmental disorders, 41(6), 741-749.

Cage, E., \& Troxell-Whitman, Z. (2019). Understanding the Reasons, Contexts and Costs of Camouflaging for Autistic Adults. Journal of Autism and Developmental Disorders, $1-13$. 
Cage, E., Di Monaco, J., \& Newell, V. (2018). Experiences of autism acceptance and mental health in autistic adults. Journal of Autism and Developmental Disorders, 48(2), 473484.

Cappadocia, M. C., Weiss, J. A., \& Pepler, D. (2012). Bullying experiences among children and youth with autism spectrum disorders. Journal of Autism and Developmental Disorders, 42(2), 266-277.

Dean, M., Harwood, R., \& Kasari, C. (2017). The art of camouflage: Gender differences in the social behaviors of girls and boys with autism spectrum disorder. Autism, 21(6), 678-689.

Engström, I., Ekström, L., \& Emilsson, B. (2003). Psychosocial functioning in a group of Swedish adults with Asperger syndrome or high-functioning autism. Autism, 7(1), 99110.

Eriksson, J. M., Andersen, L. M., \& Bejerot, S. (2013). RAADS-14 Screen: validity of a screening tool for autism spectrum disorder in an adult psychiatric population. Molecular Autism, 4(1), 49.

Faso, D. J., Sasson, N. J., \& Pinkham, A. E. (2015). Evaluating posed and evoked facial expressions of emotion from adults with autism spectrum disorder. Journal of Autism and Developmental Disorders, 45(1), 75-89.

Friedman, N. D., Warfield, M. E., \& Parish, S. L. (2013). Transition to adulthood for individuals with autism spectrum disorder: current issues and future perspectives. Neuropsychiatry, 3(2), 181-192. 
Gibson, C. M., Penn, D. L., Prinstein, M. J., Perkins, D. O., \& Belger, A. (2010). Social skill and social cognition in adolescents at genetic risk for psychosis. Schizophrenia Research, 122(1-3), 179-184.

Gillespie-Lynch, K., Brooks, P. J., Someki, F., Obeid, R., Shane-Simpson, C., Kapp, S. K., ... \& Smith, D. S. (2015). Changing college students' conceptions of autism: An online training to increase knowledge and decrease stigma. Journal of Autism and Developmental Disorders, 45(8), 2553-2566.

Grossman, R. B. (2015). Judgments of social awkwardness from brief exposure to children with and without high-functioning autism. Autism, 19(5), 580-587.

Grossman, R. B., Mertens, J., \& Zane, E. (2018). Perceptions of self and other: Social judgments and gaze patterns to videos of adolescents with and without autism spectrum disorder. Autism, 23(4), 1-12.

Halladay, A. K., Bishop, S., Constantino, J. N., Daniels, A. M., Koenig, K., Palmer, K., ... \& Taylor, J. L. (2015). Sex and gender differences in autism spectrum disorder: summarizing evidence gaps and identifying emerging areas of priority. Molecular Autism, 6(1), 36.

Hull, L., \& Mandy, W. (2017). Protective effect or missed diagnosis? Females with autism spectrum disorder. Future Neurology, 12(3), 159-169.

Hull, L., Petrides, K. V., Allison, C., Smith, P., Baron-Cohen, S., Lai, M. C., \& Mandy, W. (2017). "Putting on my best normal": social camouflaging in adults with autism spectrum conditions. Journal of Autism and Developmental Disorders, 47(8), 25192534. 
Human, L. J., Sandstrom, G. M., Biesanz, J. C., \& Dunn, E. W. (2013). Accurate first impressions leave a lasting impression: The long-term effects of distinctive self-other agreement on relationship development. Social Psychological and Personality Science, 4(4), 395-402.

Hurlbutt, K., \& Chalmers, L. (2004). Employment and adults with Asperger syndrome. Focus on Autism and Other Developmental Disabilities, 19(4), 215-222.

Hyde, J. S. (2005). The gender similarities hypothesis. American Psychologist, 60(6), 581.

Hyde, J. S. (2014). Gender similarities and differences. Annual Review of Psychology, 65, 373-398.

Iobst, E., Nabors, L., Rosenzweig, K., Srivorakiat, L., Champlin, R., Campbell, J., \& Segall, M. (2009). Adults' perceptions of a child with autism. Research in Autism Spectrum Disorders, 3(2), 401-408.

Jones, R. M., Pickles, A., \& Lord, C. (2017). Evaluating the quality of peer interactions in children and adolescents with autism with the Penn Interactive Peer Play Scale (PIPPS). Molecular Autism, 8(1), 28.

Lai, M. C., Lombardo, M. V., Pasco, G., Ruigrok, A. N., Wheelwright, S. J., Sadek, S. A., ... \& MRC AIMS Consortium. (2011). A behavioral comparison of male and female adults with high functioning autism spectrum conditions. PloS One, 6(6), e20835.

Lakens, D. (2013). Calculating and reporting effect sizes to facilitate cumulative science: a practical primer for t-tests and ANOVAs. Frontiers in Psychology, 4, 863.

Levitt, J. M. (2017). Exploring how the social model of disability can be re-invigorated: in response to Mike Oliver. Disability \& Society, 32(4), 589-594. 
Loomes, R., Hull, L., \& Mandy, W. P. L. (2017). What is the male-to-female ratio in autism spectrum disorder? A systematic review and meta-analysis. Journal of the American Academy of Child \& Adolescent Psychiatry, 56(6), 466-474.

Lord, C., Risi, S., Lambrecht, L., Cook, E. H., Leventhal, B. L., DiLavore, P. C., ... \& Rutter, M. (2000). The Autism Diagnostic Observation Schedule-Generic: A standard measure of social and communication deficits associated with the spectrum of autism. Journal of autism and developmental disorders, 30(3), 205-223.

Mattarozzi, K., Todorov, A., Marzocchi, M., Vicari, A., \& Russo, P. M. (2015). Effects of gender and personality on first impression. PloS one, 10(9), 0135529.

Matthews, N. L., Ly, A. R., \& Goldberg, W. A. (2015). College students' perceptions of peers with autism spectrum disorder. Journal of Autism and Developmental Disorders, 45(1), 90-99.

Milton, D. E. (2012). On the ontological status of autism: the 'double empathy problem'. Disability \& Society, 27(6), 883-887.

Milton, D. E., \& Bracher, M. (2013). Autistics speak but are they heard. J. BSA MedSoc Group, 7, 61-69.

Milton, D., Heasman, B., \& Sheppard, E. (2018). Double Empathy. Encyclopaedia of Autism Spectrum Disorders, 1-8.

Morrison, K. E., DeBrabander, K. M., Faso, D. J., \& Sasson, N. J. (2019). Variability in first impressions of autistic adults made by neurotypical raters is driven more by characteristics of the rater than by characteristics of autistic adults. Autism. https://doi.org/10.1177/1362361318824104 
Myles, B. S., Simpson, R. L., Ormsbee, C. K., \& Erickson, C. (1993). Integrating preschool children with autism with their normally developing peers: Research findings and best practices recommendations. Focus on autistic behavior, 8(5), 1-19.

Pellicano, L., Bölte, S., \& Stahmer, A. (2018). The current illusion of educational inclusion. Autism, 22 (4) 386-387.

Rudman, L. A., \& Goodwin, S. A. (2004). Gender differences in automatic in-group bias: Why do women like women more than men like men?. Journal of personality and social psychology, 87(4), 494.

Sasson, N. J., \& Morrison, K. E. (2019). First impressions of adults with autism improve with diagnostic disclosure and increased autism knowledge of peers. Autism, 23(1), 50-59.

Sasson, N. J., Faso, D. J., Nugent, J., Lovell, S., Kennedy, D. P., \& Grossman, R. B. (2017). Neurotypical peers are less willing to interact with those with autism based on thin slice judgments. Scientific Reports, 7, 40700.

Saxe, A. (2017). The theory of intersectionality: A new lens for understanding the barriers faced by autistic women. Canadian Journal of Disability Studies, 6(4), 153-178

Schaller, J., \& Yang, N. K. (2005). Competitive employment for people with autism: Correlates of successful closure in competitive and supported employment. Rehabilitation Counselling Bulletin, 49(1), 4-16.

Seaman, R. L., \& Cannella-Malone, H. I. (2016). Vocational skills interventions for adults with autism spectrum disorder: a review of the literature. Journal of Developmental and Physical Disabilities, 28(3), 479-494. 
Shtayermman O (2009) An exploratory study of the stigma associated with a diagnosis of Asperger's syndrome: the mental health impact on the adolescents and young adults diagnosed with a disability with a social nature. Journal of Human Behavior in the Social Environment, 19(3): 298-313

Strickland, D. C., Coles, C. D., \& Southern, L. B. (2013). JobTIPS: A transition to employment program for individuals with autism spectrum disorders. Journal of Autism and Developmental Disorders, 43(10), 2472-2483.

Taylor, J. L., \& Mailick, M. R. (2014). A longitudinal examination of 10-year change in vocational and educational activities for adults with autism spectrum disorders. Developmental Psychology, 50(3), 699.

Taylor, J. L., \& Seltzer, M. M. (2010). Changes in the autism behavioral phenotype during the transition to adulthood. Journal of Autism and Developmental Disorders, 40(12), 1431-1446.

Wood, T. J., Pugh, D., Touchie, C., Chan, J., \& Humphrey-Murto, S. (2018). Can physician examiners overcome their first impression when examinee performance changes? Advances in Health Sciences Education, 1-12.

Zaki, J. (2013). Cue integration: A common framework for social cognition and physical perception. Perspectives on Psychological Science, 8(3), 296-312. 
Table 1. Demographic Information for Observer Participants.

Percentage $(\%)$

\begin{tabular}{ll}
\hline Ethnicity & \\
White British & 54.1 \\
Other White background & 20.0 \\
Mixed / Multi-ethnic & 5.9 \\
Asian / Asian British & 17.1 \\
Black / African / Caribbean / Black British & 2.4 \\
Other & .5 \\
Sexual Identity & \\
Heterosexual / straight & 83.4 \\
Gay / lesbian & 2.0 \\
Bisexual & 11.2 \\
Don't know & 1.0 \\
Other & 1.5 \\
Prefer not to say & 1.0 \\
Education & \\
GCSEs or equivalent & 3.5 \\
Apprenticeship & 1.5 \\
A-levels or equivalent & 69.3 \\
Undergraduate degree & 16.1 \\
Master's degree & 6.8 \\
Other qualifications & 2.4 \\
Prefer not to say & .5 \\
Employment $†$ & \\
Employed Full-time & 10.7 \\
Employed Part-time & 20.5 \\
Self-employed & 1.5 \\
Unemployed & 8.3 \\
Unable to work & .5 \\
Student & 79.0 \\
Carer & 1.5 \\
Prefer not to say & 1.0 \\
\hline
\end{tabular}

†Note: Participants could select more than one option 


\section{Figure Legends}

Figure 1. Mean ratings within the video modality according to group and stimuli gender, including table with information indicating significant differences between groups. Note that awkwardness item is reverse-scored, such that a higher score indicates being perceived as less awkward. Error bars are +/- one standard error.

Figure 2. Mean ratings within the text modality according to group and gender of stimuli, including table with information indicating significant differences between groups. Note that awkwardness item is reverse-scored. +/- 1 SE bars. 

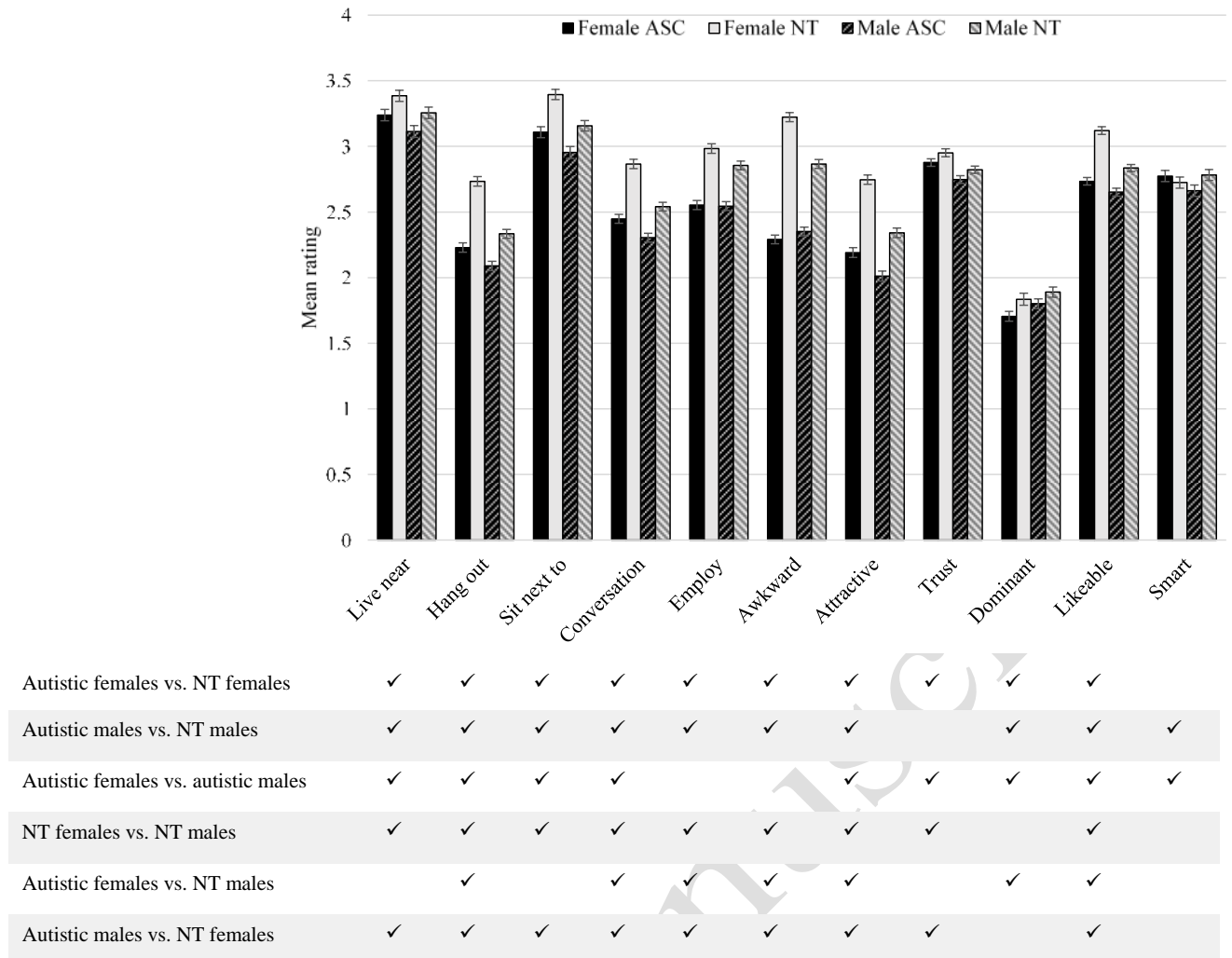

Fig 1.

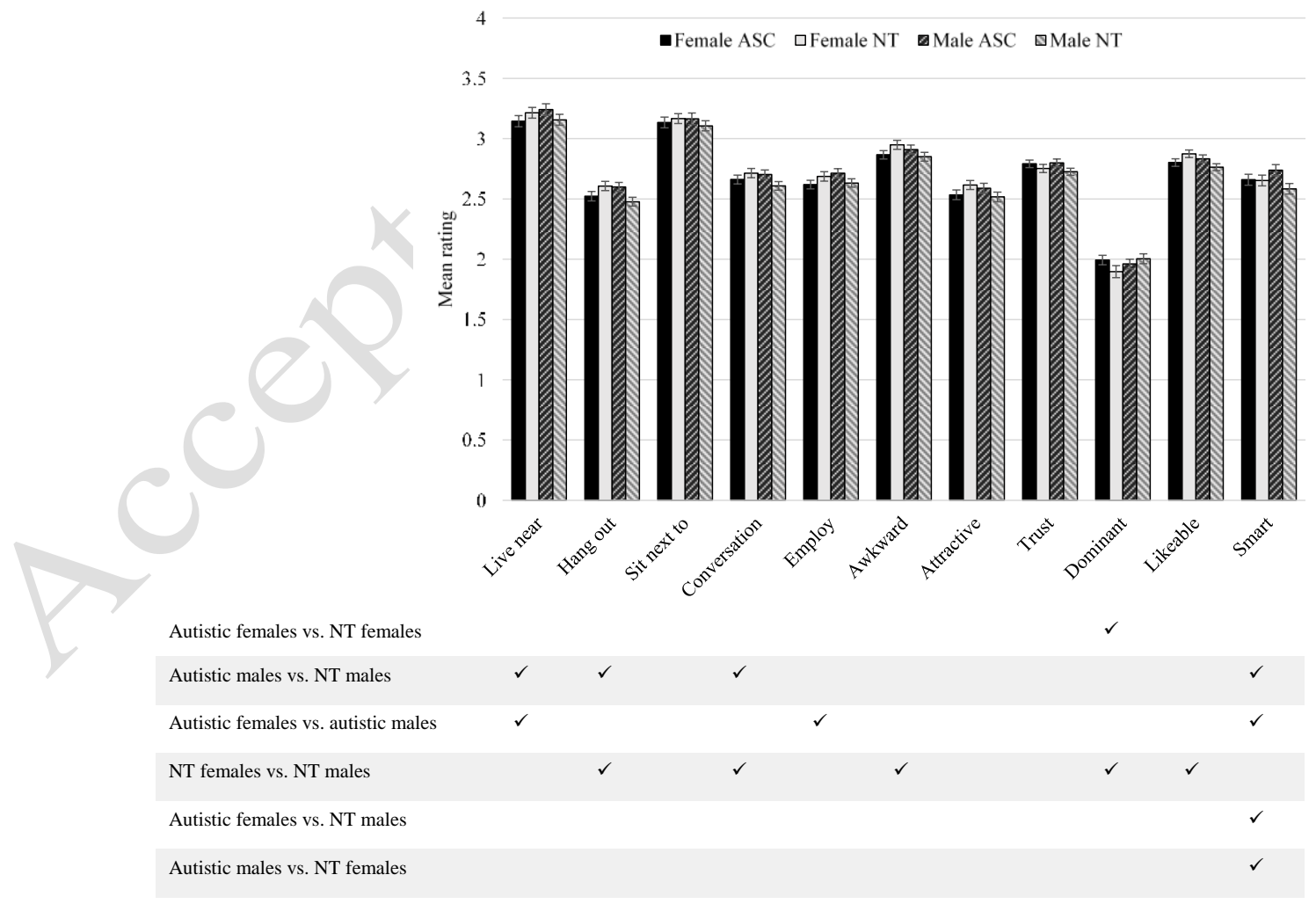

Fig 2. 